\title{
Some Remarks on Proportional Similarity
}

\author{
By J. C. GOWER \\ Rothamsted Experimental Station, Harpenden, Herts, AL5 2JQ
}

(Received 20 March 1978)

\section{INTRODUCTION}

Ware \& Hedges (1978) discussed a simple ratio-based similarity coefficient $S_{G A}$ that is certainly no worse than others in common use, including $S_{G}$ described by Gower (1971). It is not always realized how crude similarity coefficients can be. To accept a similarity scale based on ratios rather than differences is one of many subjective decisions that are constantly being taken when employing similarities. It might be appropriate to treat different characters by the different methods within the same study, or, as Ware \& Hedges (1978) suggest, if a ratio scale seems appropriate $S_{\mathrm{G}}$ can be used, with the character values logged; other transformations will be appropriate in other circumstances. Unfortunately there are few sound criteria to guide the choice between one coefficient and another. Psychological aspects of the perception of similarity are discussed by Gregson (1975).

\section{DISCUSSION}

Taking ratios eliminates problems of measurement scales providing there is a natural zero, but the advantage of $S_{\mathrm{GA}}$ in this respect is minor and with the Celsius scale, for example, $S_{G A}$ would be entirely unacceptable. Standardizing by the range, as in $S_{a}$, copes with both kinds of scale and leads to revised similarities on discovering a new operational taxonomic unit (OTU) only when new character values are outside the range of those previously allowed for. As originally described, $S_{\mathrm{G}}$ envisaged a range that could be specified larger than the sample range, so going some way towards minimizing the effects of revisions and also ensuring that 'OTUs at the extreme range do not show zero similarity' (point $e$ of Ware \& Hedges, 1978). In practice, although a zero lower value is often specified, hypothetical upper bounds are more difficult to assign and are usually ignored.

Thus $S_{a}$ complies better with Ware \& Hedges' desiderata than might appear at first sight. Even so, their assertion that 'a comparison between two OTUs should be independent of the existence or discovery of other OTUs' is questionable. A character that is constant within a set of OTUs would usually be considered inadmissible, so affecting* all similarities between pairs of OTUs of the original set. Are all constant characters therefore to be included in similarity measures to guard against the possibility of finding unsuspected states in future OTUs? This example may seem unfair, as the similarities change only because a new character is brought into play. It does, however, indicate the elusiveness of the concept of invariant similarity, a concept that seems to arise from regarding similarity as some intrinsic or absolute property of pairs of OTUs, whose value is estimated by sampling a set of characters from a universe of characters. Attractive as the idea is, the author knows of no mathematical, or other, formulation that allows the concept of intrinsic similarity to be defined, let alone have its properties explored. In practice, many classifications are determined from a set of available characters and it would be unwise to try to extrapolate to predict the effects of introducing new characters. The question, therefore, is whether similarities calculated from an assigned set of characters can justifiably change when a new OTU is discovered.

* Unless the original constant character value is 'negative' and negative matches are ignored. 
Even if a sound definition of intrinsic similarity were to be found, it seems that often one is more concerned with relative rather than absolute similarity amongst a set of OTUs. A duck may not seem very similar to a hawk, but it is when contrasted with a cat. To reflect this kind of consideration the similarity of a pair of OTUs might be required to depend on the constitution of the whole sample of OTUs, just as the similarities $S_{\mathrm{G}}$ in Table 1 of Ware \& Hedges (1978) increase after the giant OTU G has been discovered. Whether the increased similarity between duck and hawk is a consequence of accepting a wider set of admissible characters, so that the two birds share a range of negative properties that more properly pertain to cats, or is thought of as a comparison (duck, hawk) relative to the comparison (bird, cat), is hard to say. Whatever the reason, it seems likely that similarity is perceived in association with some reference set of OTUs, perhaps a class (such as birds) or a set of classes (birds, cats etc.).

In applications such as ordination, similarity or some other notion of taxonomic distance seems essential. Classification problems, however, can be formulated without appealing to the concept of similarity. For example to circumvent, or at least reduce, the difficulties referred to above, some workers prefer to dispense with similarities altogether and work in terms of predictive classifications (Gower, 1974; Barnett et al., 1975) in which OTUs are compared not with each other but with a list of properties associated with each of several classes (the class-predictors). On introducing a new OTU, the question of changes in direct inter-OTU similarity does not arise. Normally class-predictors of qualitative characters will be unaltered, so comparisons between an old OTU and the predictor of the class to which it belongs, remains as before. If disparate units, such as a sample of cat OTUs, are added to a predictive classification of birds, the effect will be to add one (or more) new cat-class; the bird-classes and their predictors are unaltered. There are, of course, instances where introducing a new OTU will tip the balance and change a class-predictor, possibly inducing some rearrangement of the classes. Unfortunately, whatever theoretical advantages this type of approach may have, computation is less well-developed and much slower than for clustering algorithms, the method is not well-suited for producing hierarchical classifications and details have been worked out only for qualitative characters. With quantitative characters much depends on how predictors are defined and how departures from predicted values are assessed. If the mode or median is used as predictor then what has been written above for qualitative characters is still valid, but if the mean is used as predictor, major disturbances may arise when a new giant OTU is introduced, and the situation is then similar to that discussed by Ware \& Hedges (1978). However, the basic point remains that predictive statements are about complete classes and not about their individual components. If a new OTU is introduced into a class then the class itself changes together, reasonably enough, with statements about the class. With maximal predictive classification (Gower, 1974), a really aberrant new OTU is more likely to fall into a new class containing only itself.

A good property of $S_{\mathrm{GA}}$, which it shares with $S_{\mathrm{G}}$, is that the resulting similarity matrix is positive-semi-definite, so ensuring a real geometrical configuration in Euclidean ordinations, using the principal-coordinate technique (see Gower, 1966). This follows from noting that if a character takes values $x_{1} \geqslant x_{2} \geqslant x_{3} \ldots \geqslant x_{n}$ in $n$ OTUs, where the assumption that the OTUs have been ranked in order of decreasing size loses no generality, then the determinant $D$ of the resulting $n \times n$ ratio-based similarity matrix is:

$$
D=\prod_{i=1}^{n-1}\left[1-\left(x_{i+1}^{2} / x_{i}^{2}\right)\right]
$$

which must be positive or zero because every term in the product is non-negative. That $D$ be non-negative is the condition that the similarity matrix be positive-semi-definite, and the same property holds for the similarity matrix obtained by averaging those obtained for each quantitative character.

The computational aspects of $S_{\mathrm{GA}}$ are less desirable than Ware \& Hedges (1978) seem to 
imply. In this kind of work, efficiency of calculation is more important than reduction of program code (point $c$ of Ware \& Hedges, 1978) as the calculation of the similarity matrix itself often accounts for most of the computer time. For quantitative characters the numerous divisions required for calculating ratios can be very expensive when calculating a large similarity matrix over many characters, even on a modern computer. To use the same method for binary characters is even worse, because if the denominator is unity, division is unnecessary and if it is zero, or unknown, special action is needed. The essential code to deal with the latter case is trivially amended to cope with the former in an efficient manner. In fact, the quantitative form of $S_{\mathrm{a}}$ also operates correctly with binary values and without the difficulties of checking for awkward divisors, but one would not take advantage of this if interested in efficiency. It seems that unordered multistate characters require special attention in $S_{G A}$, as in $S_{G}$.

The increased speed of computers conceals the fact that programming developments have actually made the calculation of similarity matrices much less efficient than they were 10 or 15 years ago. Originally the values of many binary characters could be packed into each computer word (thus saving space) and numbers of matches, either excluding or including negative matches, could be extremely efficiently evaluated using binary logical functions simultaneously operating over complete words. Perhaps similar operations will again become available in some implementations of new developments, such as Algol 68. But high level computer languages, like Algol 60 and Fortran, now in almost universal use in scientific work, do not allow such manipulations. The upshot is that to calculate a similarity matrix based on binary characters takes over 20 times as long in Fortran as in optimum machine code and the same data now occupies up to 30 times more space than when packing was feasible. Not all is loss, however. Programs are now more portable and a little easier to write, but the price paid should be recognized.

\section{REFERENCES}

BarnetT, J. A., Bascomb, S. \& Gower, J. C. (1975). A maximal predictive classification of Klebsielleae and of the yeasts. Journal of General Microbiology 86, 93-102.

GowER, J. C. (1966). Some distance properties of latent root and vector methods used in multivariate analysis. Biometrika $53,325-338$.

GowER, J. C. (1971). A general coefficient of similarity and some of its properties. Biometrics 27, 857-871.

Gower, J. C. (1974). Maximal predictive classification. Biometrics 30, 643-654.

Gregson, R. A. M. (1975). Psychometrics of Similarity. New York, San Francisco and London: Academic Press.

Ware, G. C. \& Hedges, A. J. (1978). A case for proportional similarity in numerical taxonomy. Journal of General Microbiology 104, 335-336. 